\title{
The Marriage of Community-based Participatory Research and Practice-based Research Networks: Can It Work? - A Research Involving Outpatient Settings Network (RIOS Net) Study
}

\author{
Robert L. Williams, MD, MPH, Brian M. Shelley, MD, and \\ Andrew L. Sussman, PhD, MCRP, on behalf of RIOS Net clinicians
}

Background: Interest in community-based participatory research (CBPR) has surged during the last several years because of its potential to enhance the quality and usefulness of research outcomes. Practice-based research networks (PBRNs) potentially can provide a bridge between the research endeavor and communities; PBRNs have been identified as a promising venue for CBPR. However, this marriage of CBPR and PBRNs faces many challenges, such as locus of project idea generation, time constraints, and funding limitations.

Methods: This was a case study of the application of the CBPR model and CBPR principles specific to PBRN research using a recent PBRN study of communication about traditional, complementary, and alternative medicines.

Results: Challenges (eg, added cost, time) and benefits (eg, improved research data collection process, enhanced data analysis) resulted from application of the CBPR model and are detailed.

Conclusions: For practical and ethical reasons, PBRNs need to adopt the CBPR approach for certain types, if not all, of their research. This might require operational adaptations by the PBRN (eg, a community advisory board, community membership in the network board of directors, and outreach to community groups for input into network priorities) as well as dedicated time and funding. (J Am Board Fam Med 2009;22:428-35.)

Interest in health research based in and benefitting communities has surged during the last several years. Driven in part by recognition that research processes and benefits have historically often bypassed communities, there has been a growing effort to include community members in research structures and processes. The National Institutes of

This article was externally peer reviewed.

Submitted 26 March 2009; revised 4 May 2009; accepted 5 May 2009.

From the Department of Family and Community Medicine, MSC 09 5040, 1 University of New Mexico, Albuquerque.

Funding: This publication was made possible by grant number 1 R21 AT 2323-01 from the National Center for Complementary and Alternative Medicine (NCCAM). The contents of this article are solely the responsibility of the authors and do not necessarily represent the official views of the NCCAM, or the National Institutes of Health.

Conflict of interest: none declared.

Corresponding author: Robert L. Williams, MD, MPH, Department of Family and Community Medicine, MSC 09 5040, 1 University of New Mexico, Albuquerque, NM 87131-0001 (E-mail: rlwilliams@salud.unm.edu).
Health $(\mathrm{NIH})$ has further catalyzed this interest by requiring community engagement and collaboration in research as part of its Institutional Clinical and Translational Science Award program. ${ }^{1}$ Furthermore, the NIH Director's Council of Public Representatives has articulated several key principles needed to enhance research relationships with communities, including: (1) treating the public as partner in the research process; (2) sharing research results with the community; (3) viewing research as part of a long-term commitment to the community; and (4) valuing community partnership in research. $^{2}$

These principles closely parallel those of community-based participatory research (CBPR), an approach to health research aimed at creating a mutually beneficial partnership between communities and researchers. Although a number of definitions of CBPR exist, they share the concept of a collaborative, equitable partnership throughout the 
research process. ${ }^{3-8}$ Some authors have suggested criteria by which research can be judged as fulfilling the CBPR model. ${ }^{3,8,9}$ In general, these criteria serve as benchmarks for the community participation and/or benefit at different stages of the research process.

Practice-based research networks (PBRNs), often composed of community primary care clinicians, potentially provide a bridge between the research endeavor and communities. ${ }^{5,10,11}$ Because many PBRNs focus on what works at the primary care/community level of health care, there is a natural interface between PBRNs and their members' communities. ${ }^{4,12}$ Considering this close linkage to the community, incorporation of CBPR principles in PBRN operations would seem both logical and necessary. Indeed, there have been several calls for greater incorporation of CBPR principles in primary care research of the type often conducted by PBRNs. ${ }^{4-6,9,12,13}$ However, a recent survey of PBRNs showed that less than $25 \%$ of networks include community members in a network board and less than $7 \%$ of networks work with community members as part of a network board in the review and feedback of research. There was almost no other involvement of community members in network research operations. ${ }^{11}$

This limited application of CBPR approaches by PBRNs is not surprising. ${ }^{3,13,14}$ Experience with the model more generally, beyond PBRNs, is also limited, and the feasibility of application in the PBRN setting is uncertain. Using a study of traditional, complementary, and alternative medicine (TM/ $\mathrm{CAM}$ ) in primary care as an example of the feasible application of these methods, we describe here methods for greater adoption of CBPR approaches by PBRNs. ${ }^{15,16}$

\section{Methods and Results Setting}

The Research Involving Outpatient Settings network (RIOS Net) is a New Mexico PBRN supported by the University of New Mexico. ${ }^{15,17-20}$ RIOS Net has more than 250 primary care clinicians working in predominantly underserved Hispanic and Native American communities throughout the state at community health centers, Indian Health Service sites, and academic practices.
Table 1. Key Elements of Community-Based Participatory Research ${ }^{3-8}$

- Community participation in determining research agenda

- Community participation in developing/refining research protocol

- Community participation in data collection and analysis

- Reporting research results to the community

- Community benefit from research

- Community participation in presenting research results to scientific community

\section{Common Elements of the CPBR Approach (1) Community participation in determining research agenda}

Most experts on CBPR believe that the community should have a key or even lead role in determining the priority topics for research that involves the community (Table 1). This engagement of community members before the development of research plans inverts the usual process whereby researchers develop the research topic, question, and design before approaching a community.

Example of application to a PBRN. At the time of RIOS Net's formation, a community advisory board (CAB) composed of community residents served by network members was formed. Among its roles, the $\mathrm{CAB}$ advises the network board of directors (BOD) about network priorities and specific network studies. Three members of the $\mathrm{CAB}$ are also members of the BOD, which makes final decisions about network priorities and research projects and which is otherwise composed of clinicians who are network members.

At its first annual member meeting, RIOS Net conducted a nominal group process to identify priority clinical topics on which the network would focus. Based on the results of this group process, the BOD established 7 clinical topics as network priorities. The $\mathrm{CAB}$ reviewed these topics and network staff presented the list of priorities at community meetings across the state. As a result of these discussions, community members identified 3 additional priority clinical topics that were then endorsed by the BOD. One of these network priorities was improved integration of medical care with TM/CAM care received by patients.

\section{(2) Community participation in developing/refining research protocol}

Community member participation in the development and finalization of research design and meth- 
ods helps to assure that culturally acceptable, productive, and relevant methods are used. This partnership may also help to identify improved strategies (previously unrecognized by the researchers) for implementation of the protocol in the community.

Example of application to a PBRN. In response to the TM/CAM priority topic, RIOS Net researchers proposed a project to explore and identify ways to improve the communications between clinicians and their patients about the use of TM/ CAM. Leaders of the network vetted the initial concept with community members and clinicians in the $\mathrm{CAB}$ and $\mathrm{BOD}$ and with community groups throughout the state, which led to refinement of the research question. Community meetings helped to clarify culturally acceptable limits to the research (eg, not to explore the specifics or study the effectiveness of Native American healing practices). Iterative discussions with these groups led to the development of a community- and culturally acceptable research question and protocol. NIH funding was then obtained to apply this research protocol.

\section{(3) Community participation in data collection and analysis}

It is widely agreed that one of the key benefits of the CBPR method to researchers is the collaboration with the community in data collection and analysis. Through this collaboration, community members are often able to identify more efficient and effective means for data collection and can provide insights into data interpretation that might not otherwise be accessible to the researcher.

Example of application to a PBRN. As the next step in the TM/CAM study and using a qualitative research design, RIOS Net researchers pilot tested interviews of patients regarding their communications with clinicians about TM/CAM. The interview guide produced no useful data. The researchers then consulted with focus groups of local community members in the clinic staff at each site where interviews were to take place to determine the culturally and community-appropriate way to phrase the guide. The result was a much more culturally appropriate and linguistically accurate patient interview guide and productive data collection process. With the completion of data collection, preliminary analytic findings were presented to the $\mathrm{CAB}$ for interpretation, verification, and modification or rejection, as appropriate. $\mathrm{CAB}$ members provided important context for interpreting the findings. We then presented the findings to individual patients at several clinic sites in the form of a video vignette aimed at soliciting feedback before finalizing the analysis.

\section{(4) Reporting research results to the community}

The CBPR research process is not completed until the results of the research have been shared with the communities. This dissemination process should present the results in readily understandable terms and provide suggestions as to how the results might be used in the betterment of the community's health. In turn, the community members may again provide insights through guidance in the application of results in ways that the researchers may not have considered.

Example of application to a PBRN. In addition to presenting final study results to the $\mathrm{CAB}$ and BOD, RIOS Net employs outreach/liaison staff who meet with community groups and member clinicians in their communities and practices. During these meetings, staff present the results of network research and receive feedback. These staff disseminated the findings from the study about TM/CAM communication to a variety of community groups. In the process, network investigators received guidance on next steps in the research.

\section{(5) Community benefit from research}

The partnership between the researcher and the community that is the essence of the CBPR approach implies that each party should benefit from the joint action. In addition to the obvious but often distant benefits to the community's health that result from the research, benefits to the community may come in several forms, including remuneration of community partners, training of community members, increased knowledge, donation of research equipment, or assistance with related infrastructure development or advocacy.

Example of application to a PBRN. RIOS Net assures that each community resident directly participating in a network project receives an honorarium and each group directly participating receives a gift; this policy was followed in the TM/CAM study. The network is further exploring means of supporting policy advocacy related to research findings. 


\section{(6) Community participation in presenting research} results to the scientific community

Some suggest that the partnership of CBPR should include the community's participation in presentation and publication of research results to scientific audiences. Collaboration in this aspect of the research process requires a level of individual commitment and interest on the part of one or more community members that may not be possible to sustain. However, openness to participation by community members may help to assure that the scientific products of the research maintain a focus on community relevance.

Example of application to a PBRN. A community member was coauthor of a publication resulting from the TM/CAM study in RIOS Net. ${ }^{16} \mathrm{~A}$ second publication presented additional key findings from the study. ${ }^{15}$

\section{Costs}

CBPR methods entail added costs to the research process. Those costs are both direct (eg, staff and researcher time and travel costs, remuneration of community members) and indirect (eg, greater time required to conduct the research, soliciting input, responding to suggestions).

Example of application to a PBRN. The costs to RIOS Net for the CBPR elements of the TM/ CAM study included personnel, travel, and meeting support costs aimed at partnering with community stakeholders during each phase of the project. During the development stage, investigators met with community representatives to assess interest and gather feedback. During the study, outreach staff provided updates to community members and the investigators met with the CAB. After the conclusion of the study, outreach staff disseminated results and solicited input for subsequent research activities in this area. It is important to note that costs associated with the first phase-community input in determining research agenda-were incurred before the start of the study. RIOS Net was fortunate to have other sources of funding to support outreach efforts but this may likely represent a challenge for other researchers. See Table 2 for a more detailed list of cost elements.

\section{Outcomes}

In the example presented, the outcomes of the study included a model for clinician-patient TM/ CAM communication and several scientific presen-
Table 2. Cost Categories in Support of Enhanced Community Participation in Example Research Project

\begin{tabular}{|c|c|}
\hline Cost Categories & Elements \\
\hline Personnel & $\begin{array}{l}\text { - Investigators meet with community } \\
\text { representatives and groups during } \\
\text { project development } \\
\text { - Outreach staff participate in } \\
\text { investigator meetings during project } \\
\text { development } \\
\text { - Investigators meet with CAB } \\
\text { throughout the study development and } \\
\text { operation } \\
\text { - Investigators meet with community } \\
\text { groups during data collection and } \\
\text { analysis } \\
\text { Investigator/outreach staff present } \\
\text { research results to community groups }\end{array}$ \\
\hline Travel & $\begin{array}{l}\text { Investigator and staff travel costs to } \\
\text { meet with community groups } \\
\text { throughout the course of the project } \\
\text { - CAB member travel }\end{array}$ \\
\hline Meeting Support & $\begin{array}{l}\text { - Honoraria to community members, } \\
\text { including CAB members, directly } \\
\text { participating in formation and } \\
\text { operation of the study } \\
\text { - Added telephone costs arranging } \\
\text { community meetings } \\
\text { - Associated costs of holding community } \\
\text { meetings }\end{array}$ \\
\hline
\end{tabular}

CAB, community advisory board.

tations and publications. ${ }^{15,16}$ The study revealed a set of barriers to TM/CAM communication as seen from both patient and clinician perspectives. $\mathrm{Pa}$ tients' views about clinician receptivity and initiation of discussion about TM/CAM strongly influenced patients' decisions to communicate. Patients' expectations about clinician expertise in TM/CAM were less important. Clinicians' comfort with patients' self-care approaches and clinicians' level of concern about lack of scientific evidence of effectiveness and safety of TM/CAM influenced their communication about TM/CAM with patients. Some of these barriers were unanticipated, yet most could be easily resolved by the clinician. ${ }^{15}$

\section{Discussion}

We have presented an example of how the CBPR approach can be applied to PBRN research. Consistent with current concepts about CBPR, community representatives participated directly in defining research priorities, refining the study protocol, informing data collection and analysis, and disseminating to the scientific community. Research findings have been returned to the community. Several other facets of CBPR, as defined by 
Table 3. Proposed Community-Based Participatory Research Approaches for Practice-Based Research Networks (PBRNs)

\begin{tabular}{|c|c|}
\hline Elements of Community-Based Participatory Research & Proposed Applications in PBRNs \\
\hline $\begin{array}{l}\text { Community participation in determining research } \\
\text { agenda }\end{array}$ & $\begin{array}{l}\text { - CAB } \\
\text { - Community membership in network board of directors } \\
\text { - Outreach to community groups for input into network priorities }\end{array}$ \\
\hline $\begin{array}{l}\text { Community participation in developing/refining } \\
\text { research protocol }\end{array}$ & $\begin{array}{l}\text { - Vet protocols with CAB } \\
\text { - Solicit community input through outreach contacts or through } \\
\text { relevant community groups }\end{array}$ \\
\hline $\begin{array}{l}\text { Community participation in data collection and } \\
\text { analysis }\end{array}$ & $\begin{array}{l}\text { - Engage CAB members in review of data collection plans and } \\
\text { interpretation of results } \\
\text { - Engage study participants in review/interpretation of results }\end{array}$ \\
\hline Reporting research results to the community & $\begin{array}{l}\text { - Provide report of findings to CAB, emphasizing steps to be } \\
\text { taken to use results to improve health } \\
\text { - Outreach to community stakeholder groups to report results } \\
\text { and application to improve health } \\
\text { - Report to study participants }\end{array}$ \\
\hline Community benefit from research & $\begin{array}{l}\text { - Compensation to study participants } \\
\text { - Employment/training of community members in research } \\
\text { - Assistance with community action to apply research results to } \\
\text { health improvement } \\
\text { - Increased knowledge }\end{array}$ \\
\hline $\begin{array}{l}\text { Community participation in presenting research } \\
\text { results to scientific community }\end{array}$ & $\begin{array}{l}\text { - Engage interested community member(s) or member of CAB in } \\
\text { reporting research results }\end{array}$ \\
\hline
\end{tabular}

$\mathrm{CAB}$, community advisory board.

Jones and Wells, ${ }^{7}$ were used during the project, including regular communication, project transparency, financial compensation, respect for values, accountability, and community and patient evaluation of progress.

Proponents of CBPR generally speak of several outcomes of the process, including:

- enhancing the relevance of research questions to communities at highest risk;

- enhancing the reliability and validity of measurement instruments;

- improving response rates;

- enhancing recruitment and retention;

- strengthening interventions by incorporating cultural beliefs into scientifically valid approaches;

- facilitating the effective dissemination of findings to impact public health and policy; and

- increasing translation of evidence-based research into sustainable community change.

Our example study was not designed to formally evaluate these outcomes but, as described above, we observed that the CBPR approach did fulfill several of these expectations. Although the circumstances of the specific network and study described here are unique, we believe the general concept of partnering with the community at each step of the research process is broadly feasible for most PBRNs (Table 3). We have found that such collaboration assures the relevance and appropriateness of the research questions, improves the efficiency of the data collection, enhances the data analysis, and fulfills an ethical obligation to return value to the community.

\section{PBRNs and CBPR}

Practice-based research is particularly well suited to applying CBPR methods. As Westfall et a ${ }^{11}$ point out, "there are striking parallels between PBRN research and CBPR.” Furthermore, practice-based research fits well with the vision promoted by the $\mathrm{NIH}$ Roadmap to "develop new partnerships of research with organized patient communities, community-based health care providers... who care for sufficiently large groups of patients interested in working with researchers to quickly develop, test, and deliver new interventions." 10 Strauss et $\mathrm{al}^{21}$ stress that $\mathrm{CABs}$ - one element of a $\mathrm{CBPR}$ approach that PBRNs can adopt-can "facilitate research by providing advice about the informed consent process and the design and implementation of research protocols."

There are many examples of CBPR projects in the literature but very few have been done by primary care clinicians. ${ }^{6,13}$ However, Macaulay ${ }^{13}$ calls 
for family practice physicians who do primary care research to use CBPR: "The training and skills of family physicians...make them especially suited to participatory research." The need for this is most acute in communities of "poor, vulnerable, and minority populations." Some of the specific skills family practitioners have that make them suited for conducting CBPR include established relationships, patient-centered skills, experience with uncertainty, pragmatism, and expertise with knowledge translation.

\section{Challenges of CBPR in PBRNs}

Incorporation of the CBPR model into PBRN research is not without challenges, however, as previous authors have outlined. ${ }^{5,11}$ Difficulties may arise in identifying a "community," particularly when a PBRN covers a large geographic area, and in assuring appropriate representativeness of the community. The definition of community may need to be broadened to go beyond people who simply share a geographical location to include, for example, the virtual community of patients and staff in the practices contained within a PBRN. ${ }^{4,6,7,22}$ We believe these can be addressed through a variety of mechanisms for representing the community in network operations, such as those outlined in Table 3.

A more complex challenge can be the process of matching community priorities with the scientific interest and expertise available within the network structure. In our experience, it has been helpful to set a sufficient number of priorities and for those priorities to be phrased broadly enough (eg, "problem alcohol use" or "cancer prevention") to allow a breadth of research questions to fall within the scope of those priorities. Creating partnerships with external content experts can help a PBRN address those priorities outside its existing expertise.

A cluster of challenges relates to the realignment of relationships inherent in the CBPR process. Creating the partnership with community representatives that CBPR implies requires flexibility on the part of the researcher at each step of the research process. ${ }^{4}$ This requirement for flexibility might raise concern in the researcher about his/her ability to maintain fidelity to the scientific approach and about loss of control over the research process. However, we have found that open communication with the community does not require a sacrifice of elements of the research process but can in fact strengthen the process by improving efficiency of data collection and enhancing relevance of the findings.

An extension of this partnership can lead to new views about a community's benefit from research. Traditionally, researchers have felt that the potential for individual and group benefits from the research and its findings were both ethically and practically sufficient. Some communities, however, have increasingly come to view the balance of benefits that the researcher receives (grant and salary funding, publications, career advancement) and those that patients and communities receive (often distant or minimal gain from the study procedures) as exploitative, particularly when one considers the risk that individuals or communities may assume. A new conceptualization of benefits to the community is thus called for and must include actions such as those described in item 5 (above). This new balance of benefits requires advance planning by the researcher, often together with the community, to ensure delivery and funding.

The greatest difficulty in the incorporation of CBPR methods into PBRN research is clearly the cost. Communication and outreach with community representatives and groups incur inevitable time and monetary costs that are generally not provided for in the current research funding paradigm. The majority of PBRNs are challenged to assure sufficient stable infrastructure funding to maintain basic operations; the addition of the costs for expanded communication and outreach may be difficult to support. Although there is no single solution to this problem, we believe that the increasing recognition of the importance of community engagement in the biomedical research process has begun to open up new channels for funding this engagement. $^{1}$

\section{Limitations}

Our example of the application of CBPR in a PBRN research project has limitations. As noted, the example presented is from only one network and the specific steps taken may not readily generalize to other networks. Our intent, however, is not to suggest that the specific steps described be adopted elsewhere. Rather, our intent is to demonstrate the feasibility and advantages of applying the CBPR model to PBRN research, a finding we believe is generalizable. Our observations about costs 
and benefits of applying the CBPR approach may also be specific to the example described and not generalizable; we used a more intensive model of community interaction (eg, outreach staff) than might be required in other PBRN settings to apply the CBPR model, potentially increasing our costs over those that other networks may experience.

\section{Conclusions}

In our experience, both with the example provided and with other studies in our network, adopting CBPR approaches to PBRN research requires more time than "unilateral" research, expands infrastructure costs for the network, and requires having or building good working relationships with community members and local institutions. In the example presented of a clinical study of TM/CAM communication in the primary care setting in New Mexico, however, CBPR was the most appropriate model. It was feasible and greatly enhanced the process and findings. Community and patient participation was essential in the successful conduct of the study and led to the development of a model of enhanced TM/CAM communication. Further reports on the marriage of CBPR and PBRNs could help to clarify the benefits and challenges of the relationship.

The authors gratefully acknowledge RIOS Net members, RIOS Net Community Advisory Board members, and community members whose participation/partnership in this study made it possible. We also acknowledge Melvina McCabe, MD, and Anne Macauley, CM, MD, for their helpful review of earlier versions of this manuscript. Alissa R. Segal, PharmD, and Benjamin F. Crabtree, PhD, assisted with the study described.

\section{References}

1. Department of Health and Human Services. Institutional clinical and translational science award (U54): Part I overview information. Available at: http:// grants.nih.gov/grants/guide/rfa-files/RFA-RM-07007.html. Accessed September 10, 2008.

2. NIH. Workshop proceedings: inviting public participation in clinical research: Building trust through partnerships, October 26-27, 2004. Available at: http://copr.nih.gov/reports/October_2004_COPR_ WORKSHOP_Proceedings.pdf. Accessed September 10, 2008.

3. Israel BA, Schulz AJ, Parker EA, Becker AB. Review of community-based research: assessing partnership approaches to improve public health. Annu Rev Public Health 1998;19:173-202.

4. Macaulay AC, Nutting PA. Moving the frontiers forward: incorporating community-based participa- tory research into practice-based research networks. Ann Fam Med 2006;4:4-7.

5. Green LW, Mercer SL. Can public health researchers and agencies reconcile the push from funding bodies and the pull from communities? Am J Public Health 2001;91:1926-9.

6. Macaulay AC, Commanda LE, Freeman WL, et al. Participatory research maximises community and lay involvement. North American Primary Care Research Group. BMJ 1999;319:774-8.

7. Jones L, Wells K. Strategies for academic and clinician engagement in community-participatory partnered research. JAMA 2007;297:407-10.

8. Minkler M, Wallerstein N, eds. Community-based participatory research for health. San Francisco (CA): Jossey-Bass; 2003.

9. Green LW. Guidelines and categories for classifying participatory research projects in health. Available at: http://lgreen.net/guidelines.html. Accessed October 30, 2008.

10. Westfall JM, Mold J, Fagnan L. Practice-based research-"blue highways" on the NIH Roadmap. JAMA 2007;297:403-6.

11. Westfall JM, VanVorst RF, Main DS, Herbert C. Community-based participatory research in practice-based research networks. Ann Fam Med 2006; $4: 8-14$.

12. Westfall J, Stevenson J, North American Primary Care Research Group. A guided tour of communitybased participatory research: an annotated bibliography. Ann Fam Med 2007;5:185-6.

13. Macaulay AC. Promoting participatory research by family physicians. Ann Fam Med 2007;5:557-60.

14. Wallerstein NB, Duran B. Using community-based participatory research to address health disparities. Health Promot Pract 2006;7:312-23.

15. Shelley BM, Sussman AL, Williams RL, Segal A, Crabtree BF. 'They don't ask me so I don't tell them': patient-clinician communication about traditional, complementary and alternative medicine. Ann Fam Med 2009;7:139-47.

16. Sussman AL, Rivera M. 'Be gentle and be sincere about it': a story about community-based primary care research. Ann Fam Med 2008;6:463-5.

17. Kong AS, Williams RL, Smith M, et al. Acanthosis nigricans and diabetes risk factors: prevalence in young persons seen in Southwestern US primary care practices. Ann Fam Med 2007;5:202-8.

18. Leverence RR, Williams RL, Sussman A, Crabtree $\mathrm{BF}$. Obesity counseling and guidelines in primary care: a qualitative study. Am J Prev Med 2007;32: $334-9$.

19. Sussman AL, Williams RL, Leverence R, Gloyd $\mathrm{PW}$, Crabtree BF. The art and complexity of primary care clinicians' preventive counseling decisions: obesity as a case study. Ann Fam Med 2006;4:32733.

20. Ralston S, Kellett N, Williams RL, Schmitt C, 
North CQ. Practice-based assessment of tobacco usage in Southwestern primary care patients: a Research Involving Outpatient Settings Network (RIOS Net) Study. J Am Board Fam Med 2007;20:174-80.

21. Strauss RP, Sengupta S, Quinn SC, et al. The role of community advisory boards: involving communities in the informed consent process. Am J Public Health 2001;91:1938-43.

22. MacQueen KM, McLellan E, Metzger DS, et al. What is community? An evidence-based definition for participatory public health. Am J Public Health 2001;91:1929-38. 